\title{
Tuftsin-phosphorylcholine treatment of autoimmune diseases - a benefit and a message from helminths?
}

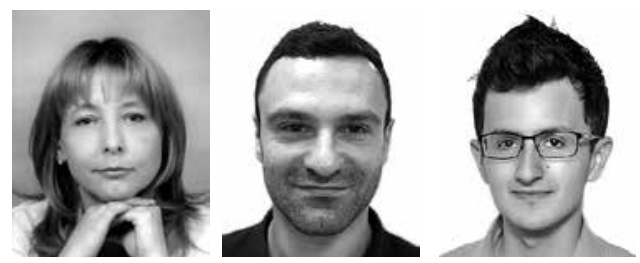

\author{
Maria Maślińska ${ }^{1}$, Fabrizio de Luca ${ }^{2}$, Kassem Sharif $3,4,5$ \\ ${ }^{1}$ Early Arthritis Clinic National Institute of Geriatrics, Rheumatology, and Rehabilitation, Warsaw, Poland \\ ${ }^{2}$ School of Medicine, Milan University, Department of Allergy and Immunology, Niguarda Ca' Granda Metropolitan Hospital, Milan, Italy \\ ${ }^{3}$ Internal Medicine B, Sheba Medical Centre, Tel-Hashomer, Israel \\ ${ }^{4}$ Zabludowicz Center for Autoimmune Diseases, Sheba Medical Centre, Tel-Hashomer, Israel \\ ${ }^{5}$ Sackler Faculty of Medicine, Tel-Aviv University, Tel-Aviv, Israel
}

The improvement of hygiene quality, especially in highly developed countries, has brought medical benefits, such as limiting the spread of infections. Yet, at the same time, this situation may create potential new threats, as we are still pondering whether contact with previously common infections can prevent or precipitate autoimmune diseases.

As we learn more and more about the importance of proper intestinal microbiome for maintaining the stability of the human immune system, it seems that in this respect even parasites infestation may have a beneficial effect. Helminths play role in inhibiting the inflammatory activity of many diseases with autoimmune background, such as inflammatory bowel disease, multiple sclerosis, type 1 diabetes, rheumatoid arthritis, and systemic lupus erythematosus [1, 2].

Studies confirmed, working mainly with animal models, that helminth infections can modulate mammalian immune response by inhibition of interferon $\gamma$ (IFN- $\gamma$ ) and interleukin 17 (IL-17) production, promotion of IL-4, IL-10, and TGF- $\beta$ release, induction of CD4(+) T regulatory cell FoxP3+ expression and generating regulatory macrophages, dendritic cells, and B cells $[1,3]$. Earlier, it was noted that in geographic areas with high frequency of worm infections fewer autoimmune diseases and atopic disorders were observed [2]. Furthermore, eradication of the infection caused an increase in the incidence of autoimmune diseases. The search for immunomodulatory effects of helminths led to the introduction of treatment with helminths and their ova in autoimmune diseases, including rheumatoid arthritis (RA), inflammatory bowel disease (IBD), and even multiple sclerosis (MS) [4].

Helminths, to survive in the host, modulate the host's immune response, so as to protect both the host and themselves from situations that overwhelm the autotolerance. This results in inhibition of inflammatory processes and autoimmune diseases. It means that by immunomodulation helminths strive to preserve the host immunobalance, i.e. its environment. The influence of microbiome disturbances on the development of autoimmune diseases has already been established; helminths also modulate the intestinal flora by moving its balance in favour of "probiotic" microorganisms [5].

It was shown that the beneficial effect of helminths is not specific to any single helminth species. The immunomodulatory effect of helminths is attributed to the phosphorylcholine (PC) molecule because the phosphorylcholine (PC)-moieties glycoproteins are secreted by helminths into the host environment. It is known that PC is presented by apoptotic cells, gram-positive bacterium (Streptococcus pneumoniae, Clostridium, Lactobacillus), and some of Gram-negative (Haemophilus influenzae) [6].

These findings led to the search for a synthetic product that can imitate the helminths' immune modulatory effect, preferably with optimal immunomodulatory effect and minimal side effects. Current studies are conducted using a phosphorylcholine (PC) conjugated with natural adjuvant tuftsin (TPC). Tuftsin is a physiological tetrapeptide (Thyr-Lys-Pro-Arg), a fraction of the IgG heavy

Address for correspondence:

Maria Maślińska, Early Arthritis Clinic National Institute of Geriatrics, Rheumatology, and Rehabilitation, Spartanska 1, 02-637 Warsaw,

Poland, e-mail: maslinskam@gmail.com

Submitted: 14.12.2017; Accepted: 18.12.2017 
chain-molecule produced by enzymatic cleavage of Fc-domain of the heavy chain of IgG in the spleen [7]. Tuftsin has been introduced into experimental disease models, such as: experimental lupus prone mice, Dextran-Sodium-Sulphate induced colitis (DSS) [8], and collagen-induced arthritis (CIA) [9]. The results of these studies turned out to be very promising - in all cases the use of TPC had a positive effect. In experimental lupus TPC delayed glomerulonephritis onset, prolonged survival time, and decreased proinflammatory cytokines such as IL-17, anti-TNF- $\alpha$, and IL-1 $\beta$ and enhanced anti-inflammatory cytokines, i.e. IL-10 and TGF- $\beta$, and triggered expression of $T$ regulatory cells [10]. Such treatment prevented the development of colitis as well [8] and reduced destruction of the joints in arthritogenic mice [9]. Treatment with TPC strongly stimulated production of anti-inflammatory cytokines, enhanced regulatory cell expression, mainly $T$ regulatory cells (but also $B$ regulatory cells), and led to the suppression of pro-inflammatory cytokines. Although the TPC treatment in lupus experimental model did not cause any significant change in total anti-dsDNA antibody concentration, glomerulonephritis activity inhibition was mainly affected by the enhanced activity of the regulatory T-cells and by the decreased activity of pro-inflammatory cytokines [10].

Summarising helminths effect: in order to survive, the helminths display the ability to increase host immune tolerance, mainly due to their influence on TLRs, inhibition of interferon production, anti-inflammatory cytokines (IL-4, IL-10) production enhancement, switching Th1 responses to Th2 (thereby inhibiting the activity of proinflammatory cytokines). They also have - by stabilising the microbiome - a beneficial effect for the host [11]. As described, the findings show that the TPC treatment is very promising. It proves that there are still many new and often surprising ways to treat autoimmune diseases to be discovered.

The authors declare no conflict of interest.

\section{References}

1. Ben-Ami Shor D, Harel M, Eliakim R, Shoenfeld Y. The Hygiene Theory Harnessing Helminths and Their Ova to Treat. Autoimmun Clin Rev Allerg Immunol 2013; 45: 211-216.

2. Okada H, Kuhn C, Feilet H, Bach JF. The "hygiene hypothesis" for autoimmune and allergic disease an update. Clin Exp Immunol 2010; 160: 1-9.

3. Elliot DE, Weinstock JV. Helminth-host immunological interactions: prevention and control of immune-mediated diseases. Ann N Y Acad Sci 2012; 1247: 83-96.

4. Smallwood TB, Giacomin PR, Loukas A, et al. Helminth Immunomodulation in Autoimmune Disease. Front Immunol 2017; 8: 453.

5. Versini $M$, Jeandel PY, Bashi T, et al. Unraveling the hygiene hypothesis of helminthes and autoimmunity: origins, pathopshysiology, and clinical applications. BMC Medicine 2015; 13: 81.

6. Weiser J. Role of Phosphorylcholine in Respiratory Tract Colonization. In: Colonization of Mucosal Surfaces, Nataro J, Cohen P, Mobley H, Weiser J (eds.). ASM Press, Washington 2005; 61-72.

7. Gao Y, Su Q, Yi Y, et al. Enhanced Mucosal Immune Responses Induced by a Combined Candidate Mucosal Vaccine based on hepatitis A virus and hepatitis E virus Structural Proteins Linked to tuftsin. PLoS One 2015; 10 (4): e0123400.

8. Ben-Ami Shor D, Bashi T, Lachish J, et al. Phosphorylcholine-tuftsin compound prevents development of Dextran-Sodium- Sulfate induced murine colitis. Implication for the treatment of human inflammatory bowel disease. J Autoimmun 2015; 56: 111-117.

9. Bashi T, Blank M. Omersel J, et al. Novel Therapeutic Compound Tuftsin-Phosphorylcholine Attenuate Collagen Induced arthritis. Arthritis Rheum 2015; 67 (suppl 10).

10. Bashi T, Blank M, Ben-Ami Shor D, et al. Successful modulation of murine lupus nephritis with Tuftsin-phosphorylcholine. J Autoimmun 2015; 5: 1-7.

11. Bashi T, Bizzaro G, Ben-Ami Shor D, et al. The mechanisms behind helminth's immunomodulation in autoimmunity. Autoimmun Rev 2015; 14: 98-104. 